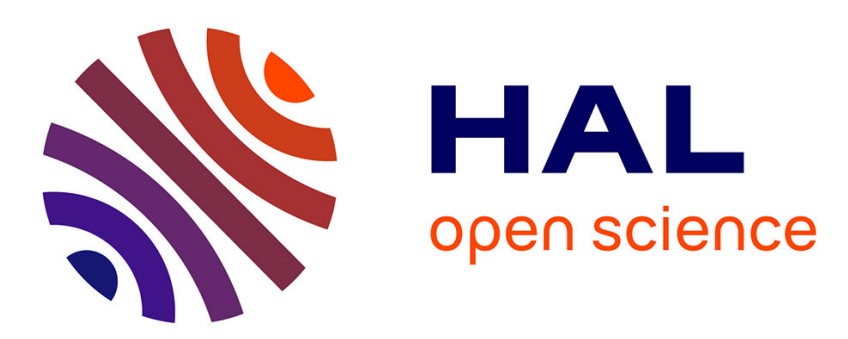

\title{
Fluids of Core-Softened Particles in Dimension Two: an Integral Equation Study
}

\author{
Aurélien Perera
}

\section{To cite this version:}

Aurélien Perera. Fluids of Core-Softened Particles in Dimension Two: an Integral Equation Study. Molecular Physics, 2009, 107 (04-06), pp.487-494. 10.1080/00268970902852657 . hal-00513274

\section{HAL Id: hal-00513274 \\ https://hal.science/hal-00513274}

Submitted on 1 Sep 2010

HAL is a multi-disciplinary open access archive for the deposit and dissemination of scientific research documents, whether they are published or not. The documents may come from teaching and research institutions in France or abroad, or from public or private research centers.
L'archive ouverte pluridisciplinaire HAL, est destinée au dépôt et à la diffusion de documents scientifiques de niveau recherche, publiés ou non, émanant des établissements d'enseignement et de recherche français ou étrangers, des laboratoires publics ou privés. 


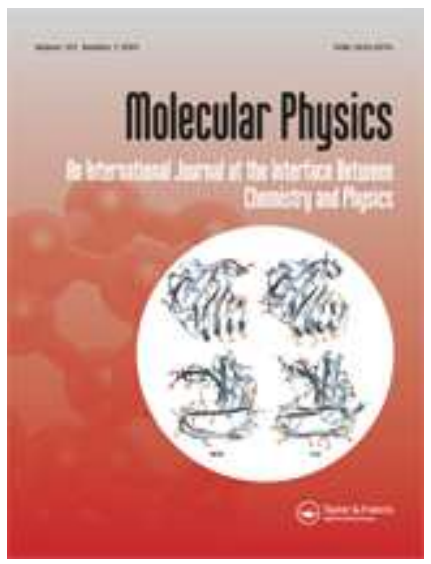

\section{Fluids of Core-Softened Particles in Dimension Two: an Integral Equation Study}

\begin{tabular}{|c|c|}
\hline Journal: & Molecular Physics \\
\hline Manuscript ID: & TMPH-2009-0040.R1 \\
\hline Manuscript Type: & Special Issue Paper - Dr. Jean-Jacques Weis \\
\hline $\begin{array}{r}\text { Date Submitted by the } \\
\text { Author: }\end{array}$ & 24-Feb-2009 \\
\hline Complete List of Authors: & $\begin{array}{l}\text { Perera, Aurélien; Université Pierre et Marie Curie, Lab Physique } \\
\text { Théorique de la Matière Condensée }\end{array}$ \\
\hline Keywords: & Liquid theory, core-softened, integral equation theory \\
\hline \multicolumn{2}{|c|}{$\begin{array}{l}\text { Note: The following files were submitted by the author for peer review, but cannot be converted } \\
\text { to PDF. You must view these files (e.g. movies) online. }\end{array}$} \\
\hline coresoft2D.tex & \\
\hline
\end{tabular}

\section{s ScholarONE" \\ Manuscript Central}




\author{
Laboratoire de Physique Théorique de la Matière Condensée (UMR CNRS \\ 7600), Université Pierre et Marie Curie, 4 Place Jussieu, F75252, Paris \\ cedex 05, France.
}

\begin{abstract}
An interaction model with core-softening, that produces clustered phases in dimension two, is studied by integral equation theories, and compared with corresponding simulation results. It is shown that the Hypernetted-chain (HNC) equation is suprizingly accurate and easier to solve numerically than the Percus-Yevick (PY) equation which appears unable to get to the cluster phase region. This is compared to the behaviour of the two theories in the absence of the core-softening: in the high temperature regime the Percus-Yevick theory is more accurate while in the low temperature regime it is the $\mathrm{HNC}$ theory that become more accurate. It is the inclusion of an infinite class of cluster
\end{abstract}


diagrams allows the latter theory to better describe phases where local structures and small clusters play a predominant role in characterizing their macroscopic properties, and the opposite behaviour observed for continuous phases of hard and soft interactions must be due to fortuitous diagram compensations in the real system. The HNC theory gives a very accurate structural description of the various cluster phases, as shown by comparing the radial distribution functions and structure factors with those from simulations.

\section{Introduction}

The rich and intriguing cluster patterns than can be formed when simple dipolar hard spheres are constrained into a bi-dimensional monolayer or bilayer have been extensively studied by Jean-Jacques Weis[1, 2, 3]. The core softening interaction model, that also leads to rich clustering, was initially introduced by Hemmer and Stell[4] to describe a particular type of liquidliquid type phase ordering, and further studied in that purpose by many authors $[5,6,7]$. The interest for this type of interaction in dimension two was recently revived by Malescio and Pellicane[8]- for the case of hard core interactions, and Camp $[9,10,11]$-for the case of soft core interactions. In both cases, it was shown that such type of isotropic interactions could lead rich variety of cluster phases. In two-dimensions, these transformations can be spectacularly visualized by snapshots: as density is increased one sees an ordinary disordered liquid, but then dimerized, striped and networked liquids. Moreover, in addition to the usual high density solid, a low density 
solid phase is also observed at very low temperatures[9]. The remarkable fact is that all these cluster "phases" are disordered: there is no Landautype one-body order parameter that can describe them. Instead, the proper statistical tools to analyze these transformations are the radial distribution function (RDF) and the corresponding structure factor (SF). The appearance of specific local structures of length scale $\xi$ leads to the emergence of specific peaks in the structure factor at wave-vector $k_{p}<k_{m}=2 \pi / \sigma$, where $k_{m}$ is the position of the main peak corresponding the diameter $\sigma$ of the particles. In contrast, at $k=0$ the structure factor is always very small, indicating that the compressibility of the liquid is small, and therefore it is not the density fluctuations that can give rise these cluster phases. This is also compatible with the fact that the corresponding core-soft interactions are repulsive.

Integral equation theory studies of core-softened interaction have been principally confined in dimension $3[13,12,14]$. The principal motivation for using core-softening seems aimed at reproducing water-like behaviour[15, 16], and eventually to relate the low temperature anomalous properties of this liquid to the possible existence of a second length scale in the interaction. This is very much along the original idea of Hemmer and Stell, since many theoreticians predict that water may have high density liquid - low density liquid phase separation in the low temperature high density part of its phase diagram[17, 18, 19]. Such studies have been also done in dimension 2[20] and also in dimension 1[21].

Recently, we have shown that many of the principal features of the radial distribution function and the structure factor of room temperature liquid water, could be reproduced in a striking fashion by two soft repulsive cores[22]. 
Moreover, we have shown that the HNC theory was able to describe this behaviour in remarkable agreement with the simulation, while the PY could not be solved for the state points of interest[22]. Despite the importance of water, the recent works $[8,9,10]$ indicate that core softening has interest beyond this single application, since it can describe the appearance of local heterogeneity within stable phases.

In the present work, we would like to examine the fate of the two diagrammatically proper integral equation closure relations, the Percus-Yevick and the Hypernetted-chain equations, when describing two-dimensional liquids with core-softened interaction. For this, we choose the same model studied by Camp, and investigate how these equations are able to deal with the appearance of clustering. The principal motivation is the following: integral equations are well known to be less reliable in the vicinity of phase transitions, where the correlations at long range put their diagrammatic insufficiency at test. Despite many investigations since almost half a century, almost nothing is known about the importance of these diagrams and the role they play. Therefore, it is of high interest to know how these approximate theories behave in the vicinity of cluster "phases".

\section{Theoretical details}

The interaction used by Camp is modeled to mimic that of dipolar spheres laying on a plane[9]. This interaction consist in a Lennard-Jones part together with a repulsive $1 / r^{3}$ part which comes from the effective dipolar part 


$$
v(r)=4 \epsilon\left[\left(\frac{\sigma}{r}\right)^{12}-\left(\frac{\sigma}{r}\right)^{6}\right]+\epsilon^{\prime}\left(\frac{\sigma}{r}\right)^{3}
$$

The parameter $\epsilon^{\prime}$ is chosen such that the resulting interaction presents an inflexion point at $r=6^{1 / 6} \sigma[9]$, leading to $\epsilon^{\prime}=8 \sqrt{6} / 9 \epsilon$.

The integral equation theory for simple liquids consists in determining the $\operatorname{RDF} g(r)$ and the direct correlation function (DCF) $c(r)$. This is achieved by solving two equations, the exact Ornstein-Zernike equation (OZ), written here in the Fourier space in terms of the structure factor $S(k)=1+\rho \int d \vec{r} \exp (i \vec{k} \cdot \vec{r})(g(r)-1)$ as[23]

$$
S(k)=\frac{2}{1-\rho \hat{c}(k)}
$$

together with an approximate closure relation, here the HNC or the PY closures[23]

$$
\begin{gathered}
g(r)=\exp \left(-\frac{v(r)}{k_{B} T}\right) \exp (g(r)-1-c(r)) \quad(\mathrm{HNC}) \\
g(r)=\exp \left(-\frac{v(r)}{k_{B} T}\right)(g(r)-c(r)) \quad(\mathrm{PY})
\end{gathered}
$$

Written as such, the last relation indicates that PY is a linearized version of the HNC closure. However, if the $\log$ of the two relations is taken, it amounts to express $c(r)$ in terms of the RDF, and it is then HNC that would appear as the linearisation of the PY equation. Diagrammatically, however, it is the HNC approximation that contains more cluster diagrams (in terms of the Mayer function $\left.f(r)=\exp \left(-v(r) / k_{B} T\right)-1\right)$, and therefore is expected 
to be more accurate. For the hard sphere fluid, the analytical solution of the PY is more accurate than the HNC equation[23]. This has led to think that the PY equation is more accurate than the HNC equation for purely repulsive interactions. The present study indicates that the presence of the infinite set of additional diagrams in the HNC equation allows to predict the local micro-structure more accurately than the PY equation. Therefore the occasional accuracy of the PY must be due to a compensation of higher order diagrams in the full theory.

\section{Results}

The integral equations have been solved on a grid of $N=1024$ points with r-step of $r=0.02 \sigma$. The reduced density is defined as usual as $\rho^{*}=(N / V) \sigma^{3}$ and the reduced temperature as $T^{*}=k_{B} T / \epsilon$. The relative weight of the core softening energy is then controlled by the parameter $\epsilon_{C}=\epsilon^{\prime} / \epsilon \approx 2.1773$. These conventions are the same as in Ref.[9], which allows a direct comparison with the reported results. The details of the methodology to solve integral equations in dimension two, and particularly the Fourier-Bessel transformation techniques needed to solve the $\mathrm{OZ}$ equation have been reported in several earlier works $[25,26,27,28]$, and will not be repeated here. In addition, we have performed constant NVT ensemble Monte Carlo (MC) simulations, with a number of particles of $N=1089$, in order to produce the radial distribution function (RDF) $g(r)$ needed for comparison with those from theory. The corresponding structure factor was obtained by direct Fourier transform of $g(r)$. Since the two-dimensional Fourier transform require log scale grid[25], 
it was necessary to interpolate the RDF obtained by simulation on a regularly spaced grid. This was done using a linear interpolation as a first approximation. The obtained results show that this crude interpolation is sufficient for a quantitative analysis. The very small $\mathrm{k}$ behaviour was however very noisy in many cases, which is due to numerical noise in the medium to long range part of the RDF obtained from the simulations, rather than the interpolation procedure itself.

\subsection{The Hard and Soft Core fluids}

In order to better assert what happens in the cases when the full coresoftening interaction is turned on, we examine the cases for $\epsilon_{C}=0$.

First, we examine the RDF from the reference hard disc fluid, for a relatively high density $\rho^{*}=0.85$, which is close to the maximum density at which the HNC closure can be solved[29]. Fig.1 shows unambiguously that the PY equation is very accurate, while the HNC equation appears to overestimate the ordering of the molecules, hence producing peaks that are shifted inward towards smaller distances -meaning tighter packing, and that are also higher than those of the MC results, indicating higher correlations. This is the reason why the HNC equation looses solutions much earlier than the PY theory, which can be solved up to higher densities. This seems equally true for hard non-spherical particles as well, where the HNC theory tends to overestimate the orientational ordering, as was verified in two[28] and three[30] dimensions.

Next, we examine the case of soft-core interactions, for three different 
temperatures, $T^{*}=5,1,0.1$, and close to the highest density that was obtainable in each case. This is shown in Fig.2(a,b,c). At high temperature (top panel), the two integral equations are comparable in accuracy, as is expected, since the interaction is depressed. At the intermediate temperature (middle panel), it is seen that the PY equation is again very accurate. This time, and very surprizingly, the HNC results appear to underestimate the correlations, as can be seen from the rapid decay of the correlations. This result indicates that the diagrams missing in the HNC theory play an important role in describing the long range behaviour of the theory. Suprizingly, the lack of diagrams in the PY theory seems to compensate each other at large distances. Finally, at the smallest temperature, it is HNC that becomes more accurate, particularly at large distances, where the PY theory shows more pronounced packing effects.

A look at the corresponding structure factors in Fig.3(a,b,c) gives interesting insights into the nature of each closure. The structure factor reveals essentially the long range behaviour of the RDF, so it gives a better insight at the large scale correlations and packing effects. For $T^{*}=5$ we learn nothing more than for the RDF in Fig.2a. For $T^{*}=1$ we see that the simulations give a second split peak that is reproduced by neither of the theories. Interestingly though, PY gives an averaged peak, while HNC fits nicely only the second part of the split peak. As to the first peak, both MC and PY reach about 8 while HNC reached only half that value. From the third peak on, the agreement between PY and MC is perfect. The second split peak indicates the existence local crystalline order within the second neighbour shell. At the smallest temperature (lower panel) we see that the differences between 
the two theories is less obvious, and it is the PY theory that would seem to better fit the overall features of MC data, except for the second peak again. So, the good agreement observed in the RDF between HNC and MC for this case seem to stem essentially from the second peak feature of the $S(k)$.

A look at the snapshots for the temperature $T^{*}=1$ indicates that the system looks glassy like, with predominance for local semi-crystalline heterogeneity, which explain the feature in the second peak of $S(k)$, of which HNC seems to capture only some of the features. For the lowest temperature, it is also HNC that has solution for higher densities (up to $\rho^{*}=0.6$, while PY stop having solutions at $\rho^{*}=0.51$.

The main conclusion of this part is that, while PY may seem a better theory for purely repulsive cores, the secondary structure is better captured by a richer diagrammatic theory. There are intriguing compensation effects in the PY theory that one is eager to know the origin of.

\subsection{The core-softened fluid}

\subsubsection{The phase diagram}

The phase diagram obtained by the two theories is shown in Fig.4, and compared to that taken from the simulations of Ref.[9]. As usual, it is the limit of solution of the two theories that are obtained, and not the actual "phase" boundaries. The first remark is that either theories give a single boundary, where the simulations of Ref[9] indicate the existence of four phases: two solid phases, one at high density (S) and one a low density and low temperature (LS), one cluster phase (C) and the usual fluid phase (F). The second 
remark is that either integral equations have only a "fluid" phase that terminates at a boundary with is very different for each of them. In particular, we note that HNC has a boundary very close to the S phase, while that of PY is well within the $\mathrm{F}$ phase. Both theories have no solutions to the right of their respective boundaries. The first conclusion is that the PY theory cease to have solutions at very small densities, while the HNC theory has solutions well inside the $\mathrm{C}$ phase region. This situation is already very different from that found in the hard and soft core cases where both theories could be solve quite close to the fluid-solid boundary, except precisely at very low temperatures, where the PY theory starts to have difficulties to handle high densities. So, we expect that the explicit clustering observed here is the reason why PY cannot solved even deep inside the $\mathrm{F}$ phase where no clustering is observed. This fact alone indicates that PY theory is diagrammatically insufficient.

Fig. 5 shows how the core-softened interactions vary as the temperature is lowered. Thus, at very low temperatures, the particles see mostly the outer repulsion, except at very high packing where they need to surmount this barrier in order to reach close packing. This explains why a low diagrammatic theory might be able to describe properly such configurations that require cooperative coordination of a large ensemble of particles.

The very low temperature region below $T^{*}=0.003$ could not be reached by both theories for numerical reasons. Indeed, the interaction at the core part becomes very repulsive, and they tend to dominate the effective part of the interactions that come from the correlations between molecules, and which give rise to the clustering. It is probably possible to explore this region by using some reference trick to eliminate the numerical barrier due the huge 
repulsion, but we have not tried this at this stage.

\subsubsection{Structural properties}

First, we report in Fig.6 a comparison of the structure between the simulations and the two theories at two state points in the fluid phase, one at high temperature $T^{*}=1$ and $\rho^{*}=0.8$ and the other at a lower temperature $T^{*}=0.05$ and $\rho^{*}=0.1$. For $T^{*}=1$ there is no cluster phase yet, even at density as high as $\rho^{*}=0.8$, and the theories behave similarly to the previous case, with PY being clearly more accurate. At high temperature, the softening barrier is very low, so all particles can pack up to the smallest core. At lower temperature, and at fluid phase density, the softening barrier is relatively high, and all particle pack up to this distance. Both these features are well reproduced by both theories. However, at $T^{*}=0.05$, the small peak at $r \approx \sigma$ is more apparent for HNC, indicating that this theory sees that some particles can still come close to main core. The PY theory fails when the density is high enough so that some particles need to get close to $r=\sigma$. Moreover, the oscillations of the RDF are wider, indicating that this theory mostly sees the outer energy barrier. This could explain why it ceases to have solutions at lower densities: it is because these correspond to an effective larger packing fraction corresponding to a larger particle size. This example proves directly the diagrammatic insufficiency of PY. On the contrary, HNC is very accurate.

Fig.7 shows a sequence of RDF as $\rho^{*}$ is varied at fixed temperature $T^{*}=$ 0.01. Fig. 8 shows the corresponding structure factors. Fig.9 shows the snapshots corresponding to the four densities. The first $\rho^{*}=0.05$ is in the very 
low density fluid phase, for which PY can still be solved. The second $\rho^{*}=0.2$ is well inside the fluid phase, in the region where dimers and some trimers are observed. The third $\rho^{*}=0.3$ is in the curled stripes cluster phase. The last $\rho^{*}=0.45$ is in the networked cluster phase. The first case in the only one that contains a PY solution. Much like in Fig.6, one can see that PY exaggerates the packing due the outer core, showing again its diagrammatic insufficiency. This is seen both by the wider oscillations in the RDF and the high and narrow first peak at $k \sigma \approx 2 \pi / 5$ in the SF. Deeper in the F phase, where dimers appear, HNC is able to both track this formation by showing a first peak in the RDF at $r=\sigma$ and in good agreement with MC. The large second peak indicates that most particles are still repelled by the outer core. The agreement with MC is out of phase after these two first peaks, indicating that HNC has some important diagrammatic insufficiency which may be able to both describe entropical aggregation and loose particles. In the cluster phase, however, the agreement become excellent, both in $\mathrm{r}$ and $\mathrm{k}$ spaces. First, the structural signature of the stripe phase at $\rho^{*}=0.3$ is well captured. This is particularly seen in the SF representation, where the main peak at $k \sigma \approx 2 \pi$ due to the contact at $r=\sigma$ is of equal height than the pre-peak at $k \sigma \approx 2 \pi / 2$ that is due soft repulsion at $r \approx 2 \sigma$. This equality of peaks and pre-peak height illustrates very well the perfect symmetry breaking that occurs in the effective interactions in the stripe phase. Indeed, each particle is exactly in the two configurations imposed by the two length scales that appear in the interaction energy, one at $r=\sigma$ and the other at $r \approx 2 \sigma$. The fact that an isotropic interaction leads to an effective anisotropic one is very remarkable. Finally, the structure of the networked fluid is equally 
well captured at $\rho^{*}=0.45$, but the corresponding features are harder to read from the RDF and the SF.

It is puzzling that HNC performs so well for well morphed cluster phases, but not so for the dimer-trimer region which at a lower density. This is certainly an indication about its diagrammatic structure and would deserve further investigations.

Finally, we note that the HNC equation is numerically solved very easily, even very near the no-solution regions, as the iteration cycles converge very rapidly. In contrast the PY theory appears very difficult to converge and larger number of iterations are often required. This is in contrast also to what happens when the second soft repulsion is absent, where both theories are sluggish near the fluid-solid phase boundary. The feature noted above is important if put in relation with the cluster nature of these theories.

\subsubsection{Phases and boundaries}

Integral equations are notoriously known for their deficiencies near phase boundaries[31]. These deficiencies are usually related to the appearance of long range correlations due to enhanced density fluctuations and the subsequent growth of the correlation length. However, such behaviour is usually related to presence of attractive part of the interaction. In the present case, and in the absence of attractive interactions, the $k \rightarrow 0$ behaviour of the structure factor is never anomalous, both in the simulations and the integral equation results, which is to say that the compressibilty of these liquids is always smal. Rather, it is the presence of the pre-peak that witness the appearence of clusters instead of large scale density fluctuations that could 
contribute at $k=0$. The fact that HNC is able to predict the appearance of the clusters phases, when it is not capable of describing properly long range correlations, is both noteworthy and intriguing. Indeed, it is often thought that integral equations can be solved only until the stability limit of the phase they are solved for. The results shown here would then tend to suggest that the various cluster phases observed here are not true thermodynamical phases. This conclusion, drawn from an approximate integral equation result, is in variance with the second order stripe phase transition reported in Ref.[8], albeit for a different model. Similar consideration holds equally for the low density solide phase reported in Ref.[9] which is not found here for the same temperature and density range. Such conclusion might be, at first, enforced by the fact that integral equation theories are solved in the true thermodynamical limit, while computer simulations suffer from inevitable finite size effects that might affect the true periodicity of a phase. However, one should be cautious, because the thermodynamical inconsistency of the HNC theory might hinder its ability to describe proper phase transition as such. In particular, the boundaries of the phase related to the HNC approximations might be shifted towards lower temperatures, which are not currently reachable. Nevertheless, the present findings are intriguing in the sense that the structural and thermodynamical agreement between the theory and the simulations leave open the question about the thermodynamical nature of cluster phases. 


\section{Conclusion}

The previously analyzed core-softened interaction of Ref[9] is re-explored within the well known diagrammatically proper integral equation theories such as the PY and the HNC closures. The analysis reveals that the cluster based diagrammatic origin of these approximations shows their respective efficiency. These are masked when describing continuous phases. Hence it is often difficult to conclude that a lower diagrammatic theory is better if it compares well with simulation results. This is very much similar to the situation with mean field theories that can often better describe physical situations that those that incorporate higher order fluctuations. This is the reason why integral equation theories are so difficult to solve near phase boundaries where fluctuations are important. The same reason also indicates that a good structural agreement is not necessarily a sign that a better description is obtained. This comment particularly concerns the various diagrammatically improper closures, that have become so popular since they allow a better fit of the properties and structure of various systems, and that are more often used in place of diagrammatically proper closures[13]. The fact that the HNC equation is so adapted to describe cluster "phases" clearly indicates its diagrammatic superiority. In the present analysis, the absence of the so-called bridge cluster diagrams do not seem to play an important role as they do in continuous phases. The absence of attractive interaction induced fluctuations is also important here, and may also influence the fate of these methods. Further investigations into the success and failure of these model interactions is certainly needed to achieve a better understanding and 
improving integral equation theories.

\section{Acknowledgment}

The author is pleased to contribute to the Festchrift in honor of Jean-Jacques

Weis. Stimulating discussions with Philip Camp are also acknowledged.

\section{References}

[1] J. J. Weis, Mol. Phys. 100, 579 (2002)

[2] J. J. Weis, J. Phys.: Cond. Mat. 15, S1471 (2003)

[3] J. J. Weis, Phys. Rev. E77, 051501 (2008)

[4] P. C. Hemmer and G. Stell, Phys. Rev. Lett. 24, 1284 (1970)

[5] Stell and P. C. Hemmer, J. Chem. Phys. 56, 4274 (1972)

[6] G. Stell, and C. K. Hall, J. Chem. Phys. 65 , 2161 (1976

[7] M. Kincaid and G. Stell, J. Chem. Phys. 67, 420 (1977)

[8] G. Malescio and Pellicane, Nat. Mater. 2, 97 (2003).

[9] P. J. Camp, Phys. Rev., E 68 , 061506 (2003) .

[10] P. J. Camp, Phys. Rev., E 70 , 031507 (2005).

[11] P. J. Camp, J. Mol. Liq. 127, 10 (2006)

[12] G. Malescio and G. Pellicane, Phys. Rev. E63, 020501R (2001) 
[13] E. Lomba, N. G. Almarza, C. Martin and M. McBride, J. Phys. Chem. 126, $244510(2007)$

[14] N. Choudhury and S. Ghosh, Phys. Rev. E66, 021206 (2002)

[15] E. A. Jagla, Phys. Rev. E58, 1478 (1998)

[16] E. A. Jagla, J. Chem. Phys. 111, 8990 (1999)

[17] S. V. Buldyrev, P. Kumar, P. G. debenetti, P. J. Rossky and H. E. Stanley, PNAS 104, 20117 (2007)

[18] P. Kumar, S. V. Buldyrev, F. Sciortino, E. Zaccarelli, H. E. Stanley, Phys Rev E 72, 021501 (2005)

[19] A. B. de Oliveira, P. A. Netz and M. C. Barboza, Eur. Phys. J. B34, $481(2008)$

[20] A. Scala, M. R. Sadr-Lahijany, N. Giovambattista, S. V. Buldyrev and H. E. Stanley, Phys. Rev. E63 , 041202 (2001)

[21] M. R. Sadr-Lahijany, A. Scala, S. V. Buldyrev and H. E. Stanley, Phys. Rev. E60 , 6714 (1999)

[22] A. Perera, A. Rispe, L. Zoranic, R. Mazighi and F. Sokolic (to be published)

[23] J. P. Hansen and I. R. McDonald, Theory of Simple Liqu1968)ids (Academic, London, 1986).

[24] F. Lado, J. Chem. Phys. 49, 3092 (1968). 
[25] J. D. Talman, J. Comput. Phys. 29, 35 (1978).

[26] J. M. Caillol, D. Levesque and J. J. Weis, Mol. Phys. 44, 733 (1981).

[27] F. Lado, Mol. Phys.63, 623 (1988)

[28] P. G. Ferreira, A. Perera, M. Moreau and M. M. Telo da Gama, J. Chem . Phys. 95, 7591 (1991)

[29] F. Lado, J. Chem. Phys. 49, 3092 (1968).

[30] A. Perera, G. N. Patey and J. J. Weis, J. Chem .Phys. 89, 6941 (1988).

[31] L. Belloni, J. Chem. Phys. 98, 8080 (1993) 


\section{Figure captions}

Fig.1 (color online) The RDF for the 2D hard disc fluid at $\rho^{*}=0.85$. Simulation data in green, HNC in blue and PY in magenta. The inset is a closeup at the first peak.

Fig.2 (color online) The RDF of the $2 \mathrm{D}$ soft sphere fluid at $\left(T^{*}=\right.$ $\left.5, \rho^{*}=0.8\right)($ top panel $),\left(T^{*}=1, \rho^{*}=0.8\right)$ (middle panel), $\left(T^{*}=\right.$ $\left.0.1, \rho^{*}=0.5\right)$ (lower panel). The color conventions are the same as in Fig. 1

Fig.3 (color online) The structure factors corresponding to the RDF in Fig.2

Fig.4 (color online) The phase diagram of the 2D core-softened fluid. That from the simulations is reproduced from Ref.[9]. The blue symbols represent the solution boundary obtained for the HNC theory, and the magenta symbols for PY. The lines through the symbols are indicative.

Fig.5 The 2D core-softened interaction energy for three different temperatures $T^{*}=0.1,0.05,0.01$ labeled on the corresponding curves.

Fig.6 (color online) The RDF for the core-softened fluid for state points $\left(T^{*}=1, \rho^{*}=0.8\right)\left(\right.$ top panel) and $\left(T^{*}=0.05, \rho^{*}=0.1\right)$ (bottom panel). The color conventions are as in Fig.1

Fig.7 (color online) The RDF for the core-softened fluid for $T^{*}=0.01$ at four different densities la belled on corresponding panels. The 
color conventions are as in Fig.1

Fig.8 (color online) The structure factors corresponding to the RDF shown in Fig.7, with the same color conventions.

Fig.9 (color online) The snapshots for the state points corresponding to the RDF shown in Fig.7. 


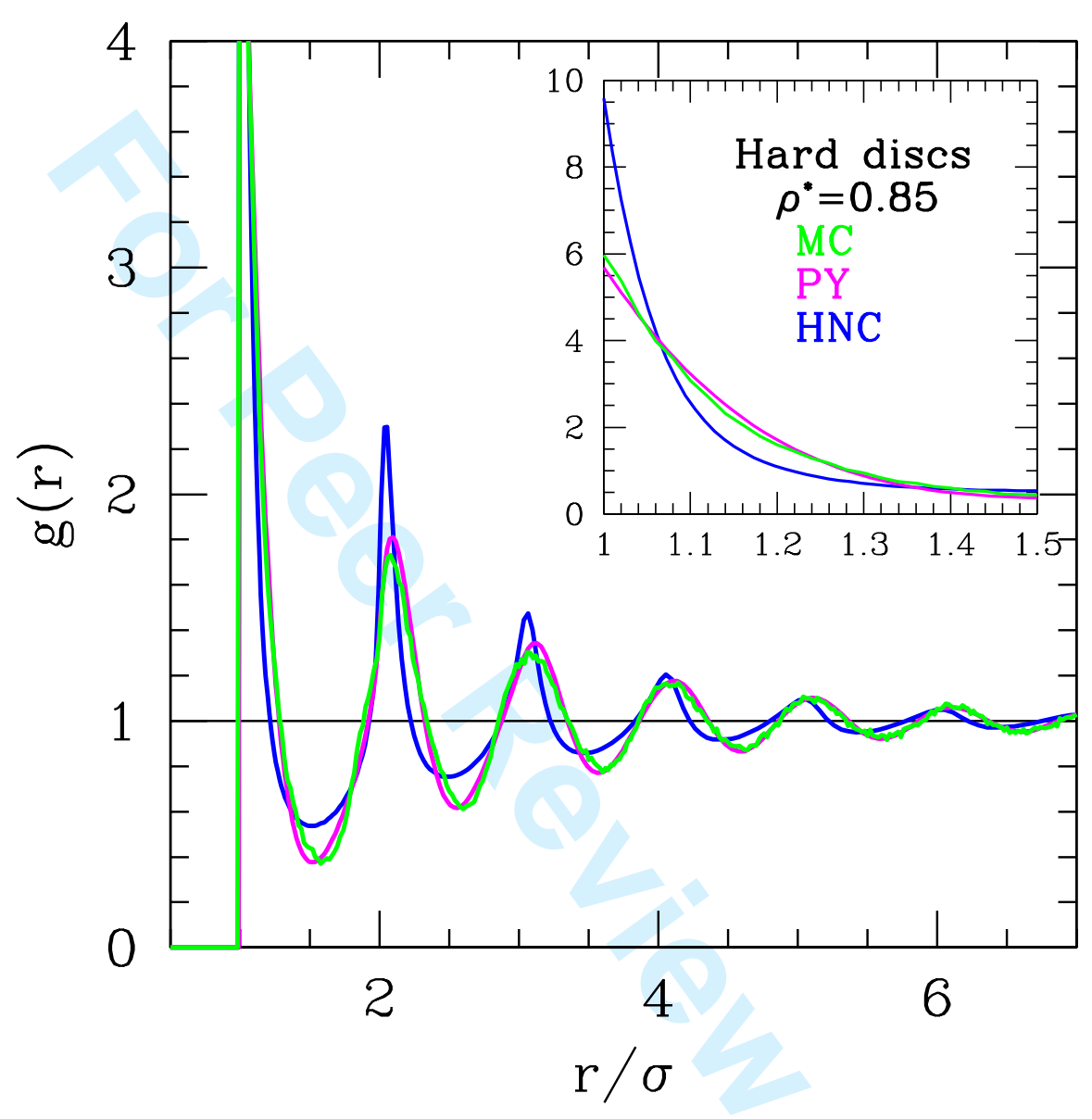




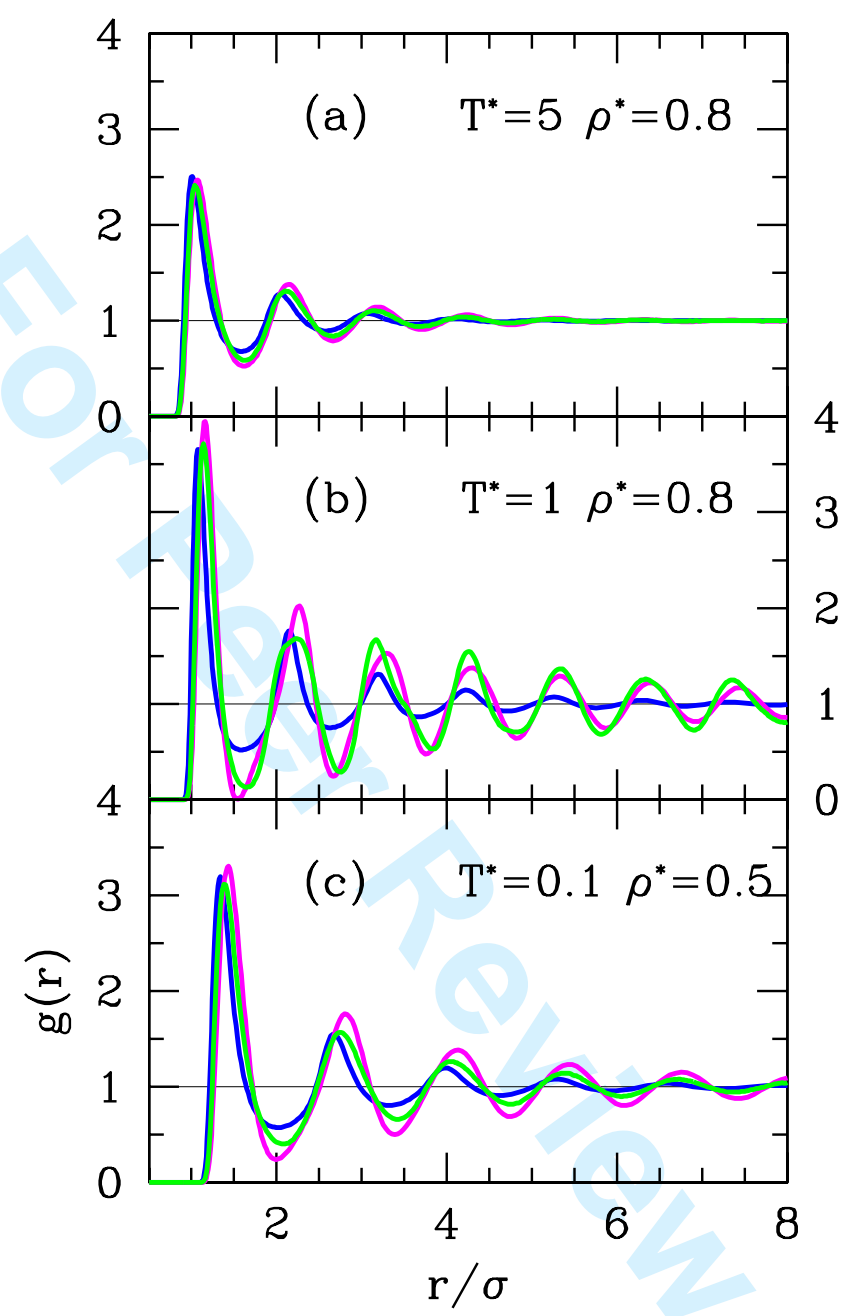




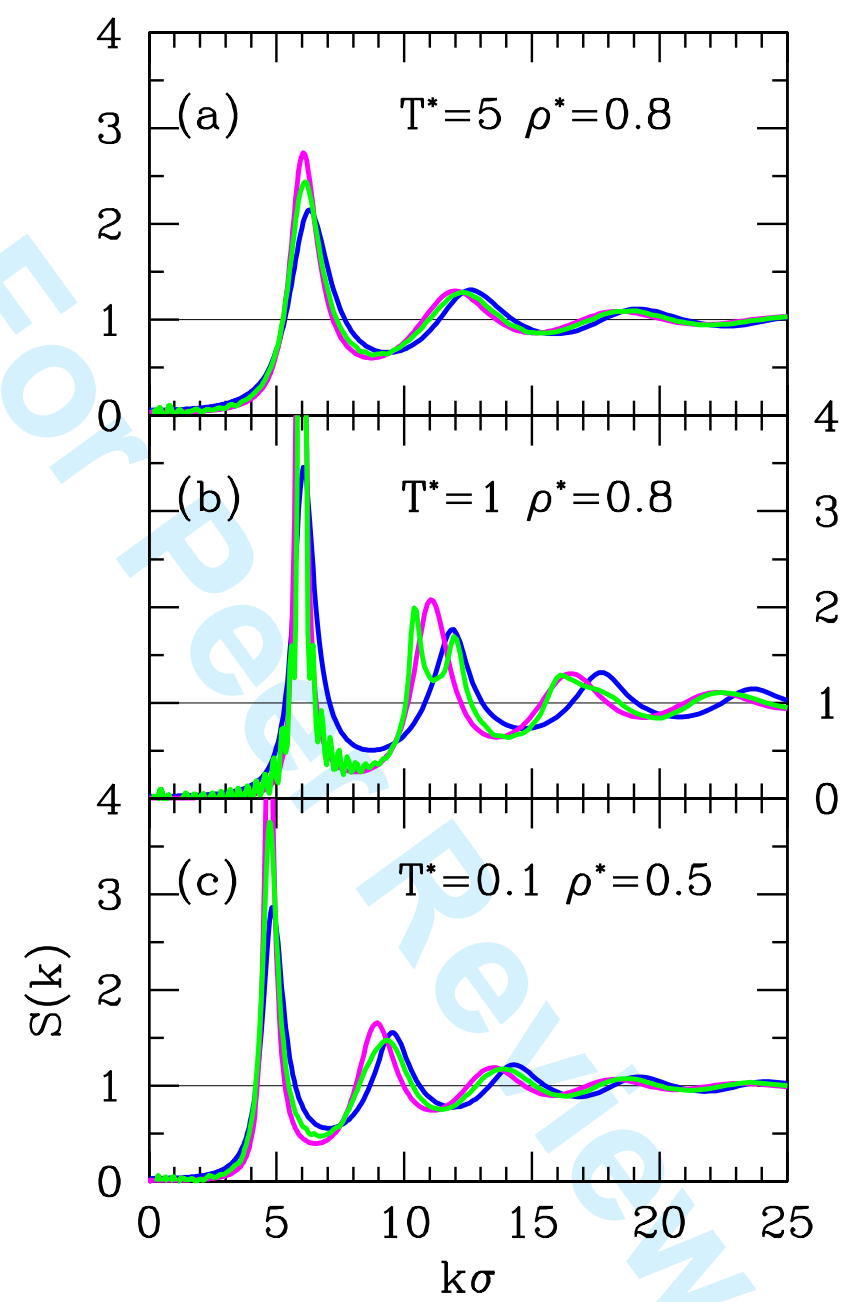




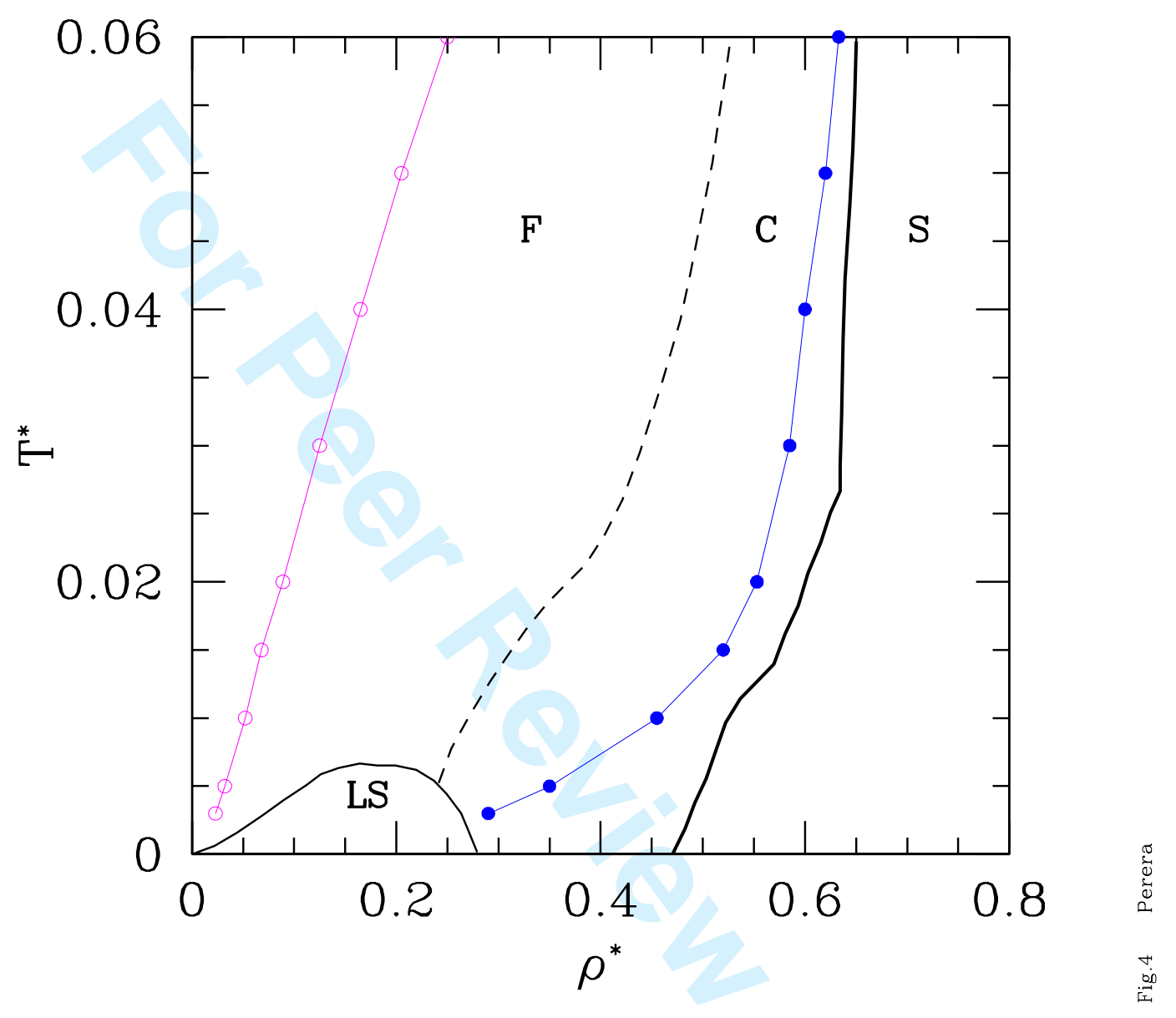




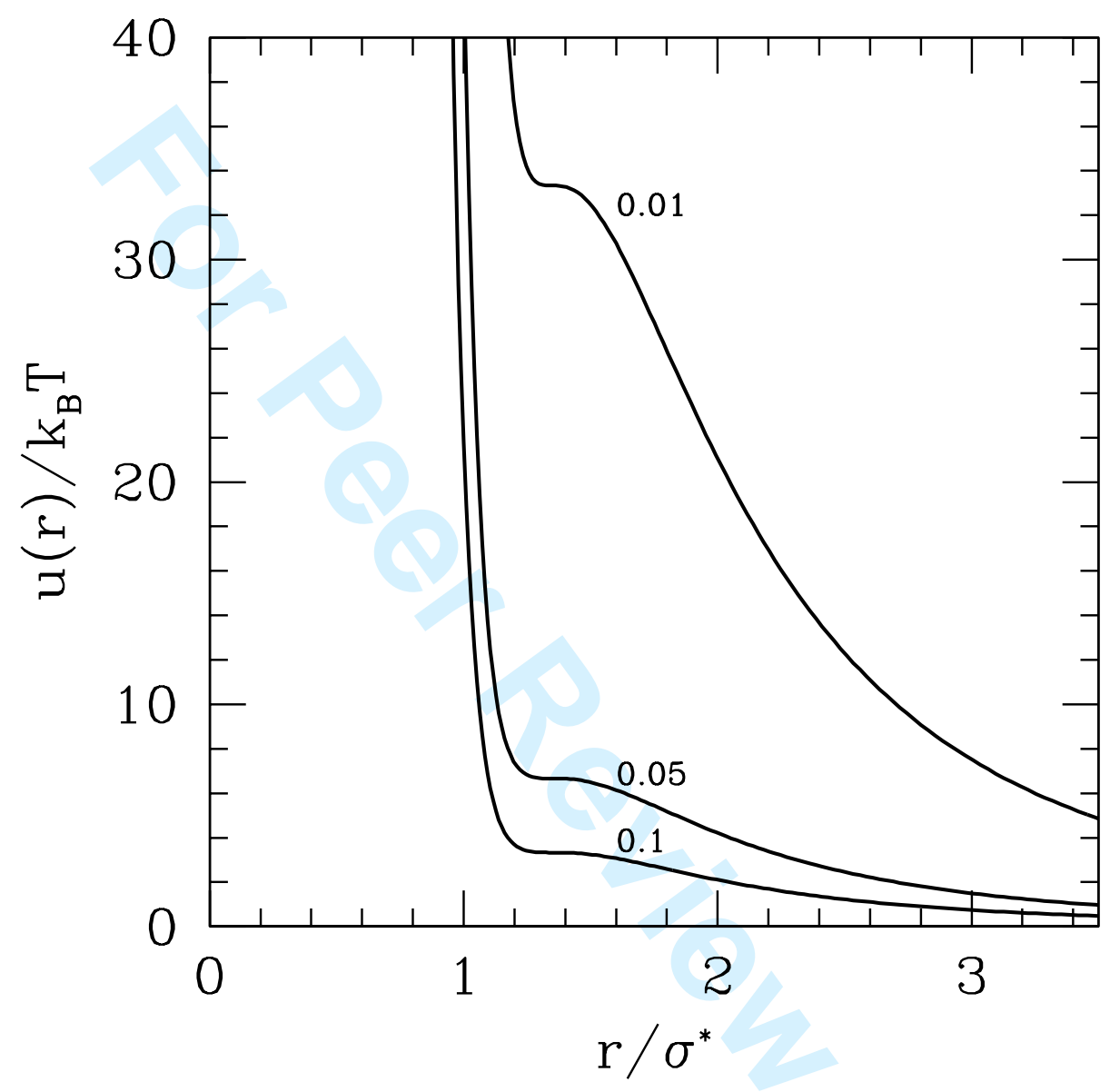




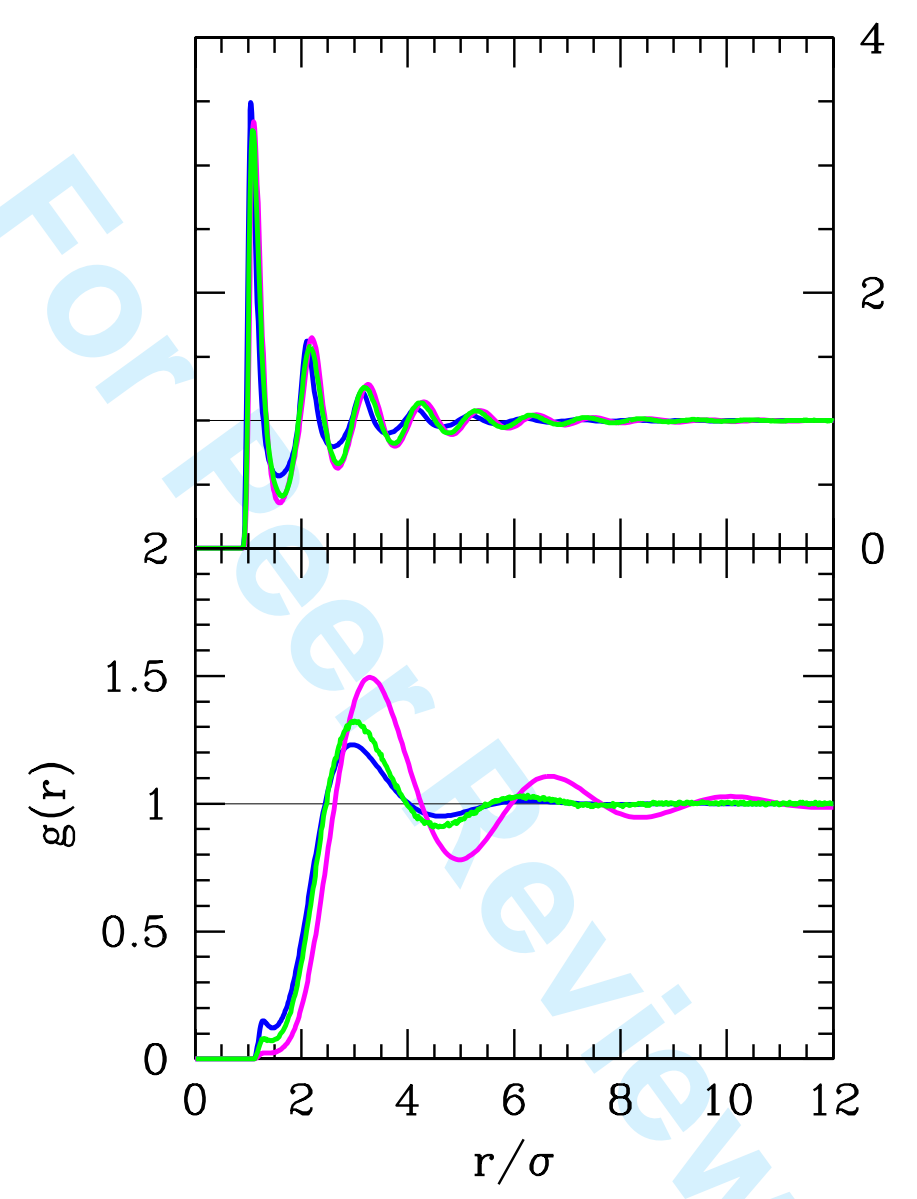




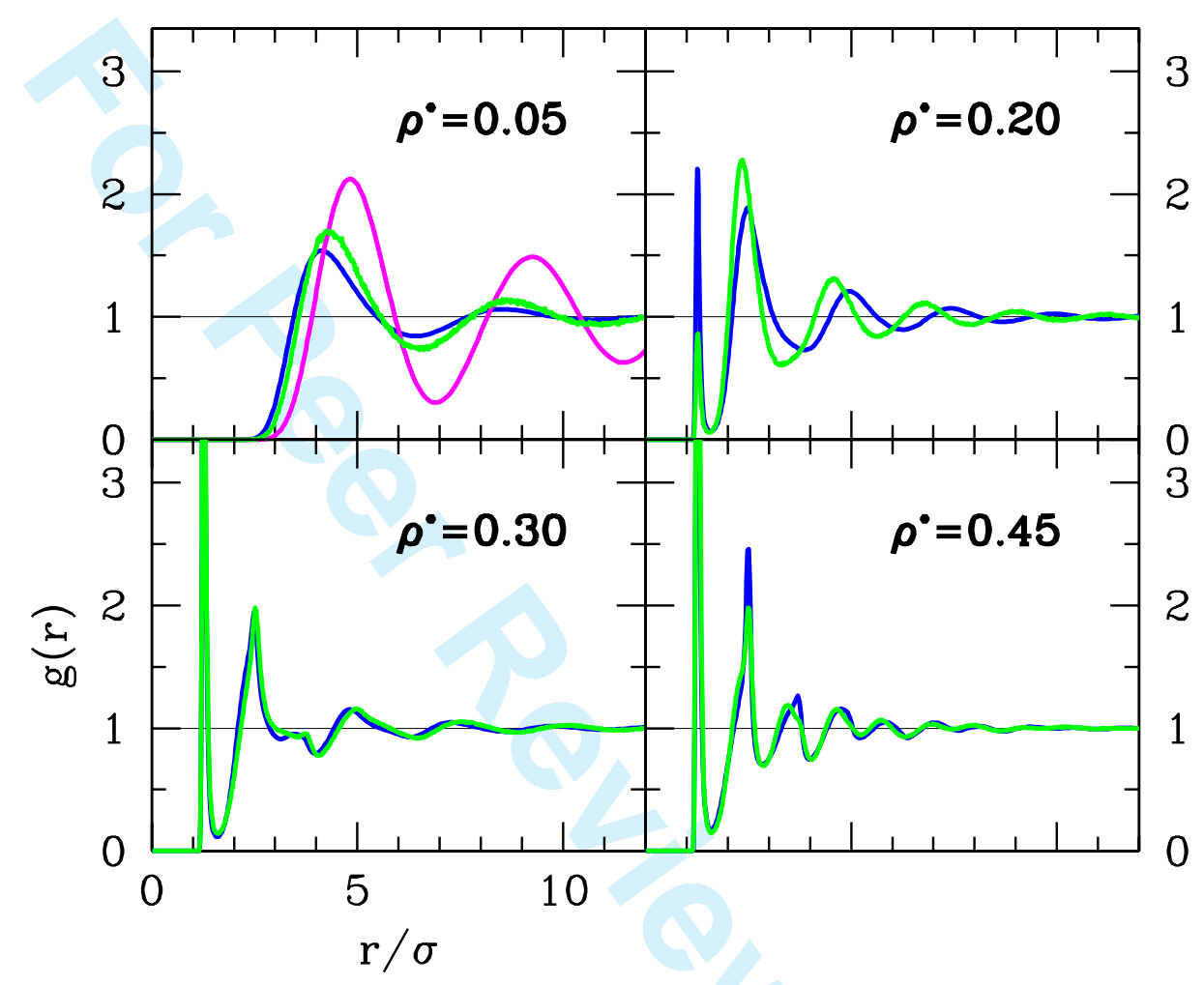




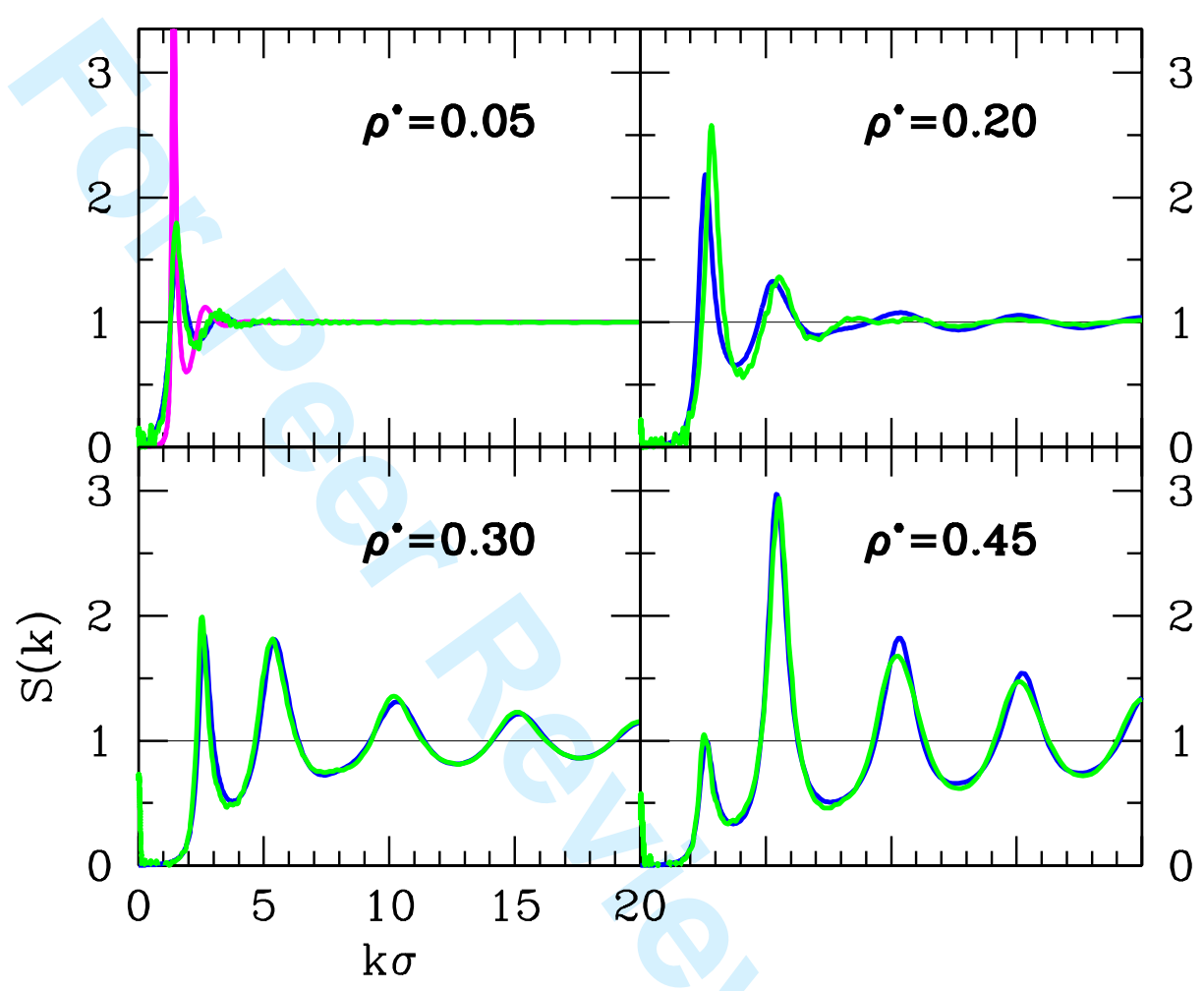




$$
\rho^{*}=0.05
$$

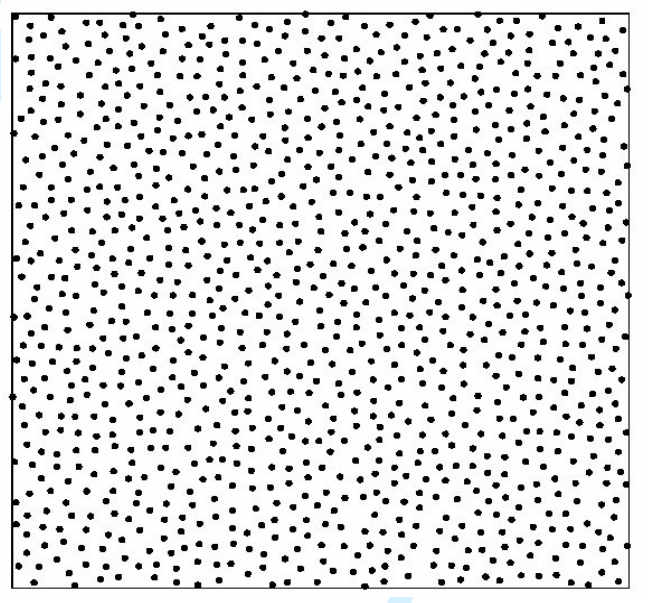

$$
\rho^{*}=0.3
$$

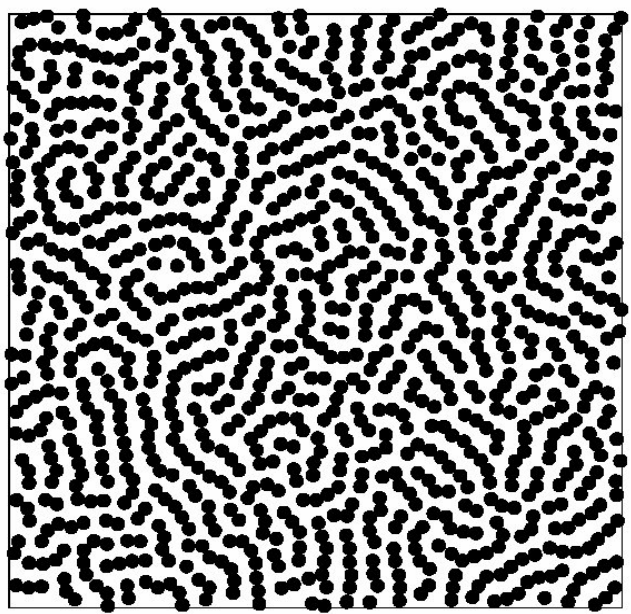

$$
\rho^{*}=0.2
$$

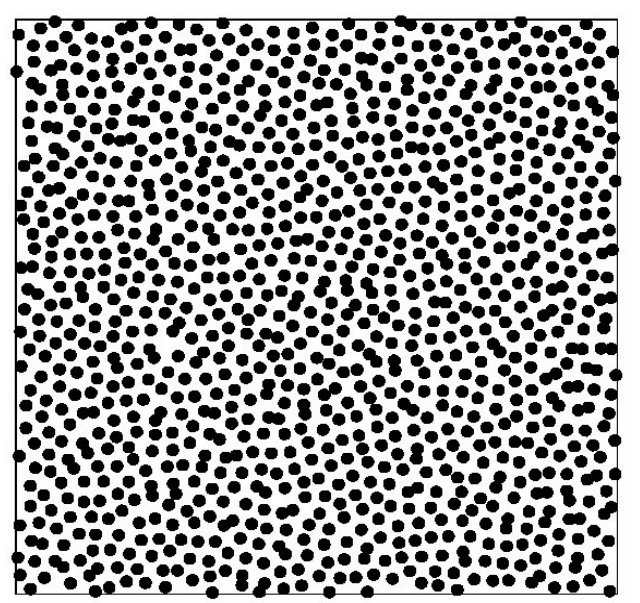

$$
\rho^{*}=0.45
$$

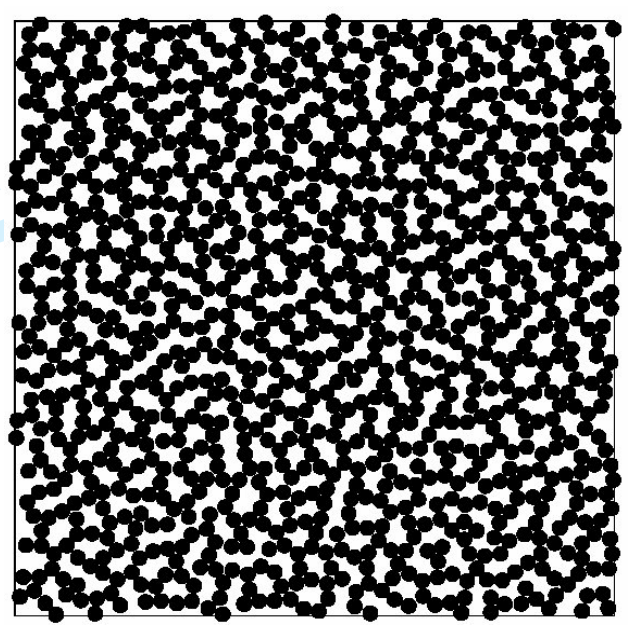

